\title{
ESTILOS DE VIDA SALUDABLE Y CALIDAD DE VIDA EN LA COMUNIDAD UNIVERSITARIA ${ }^{1}$
}

\section{Luis Valenzuela*}

\section{RESUMEN:}

El artículo se encuentra en el contexto de la Tesis Doctoral Factores de Riesgo de la Salud en Estudiantes de Pedagogía en Universidades Chilenas, y expone la importancia de cuidar por los estilos saludables y la calidad de vida de las personas en un escenario formativo, del significado de una universidad saludable en la formación de profesores, Como así también de la responsabilidad social que tienen estas instituciones en que sus estudiantes desarrollen las competencias necesarias para enfrentar los problemas de salud, el mejoramiento de la calidad de vida y la carencia o insuficiencia de hábitos y estilos de vida saludable en la población universitaria del sistema educacional chileno en la actualidad.

Palabras clave: Estilos de vida saludables, Calidad de vida, Universidad saludable, rol del profesor.

\section{HEALTHY LIFE STYLES AND LIFE QUALITY IN THE UNIVERSITY COMMUNITY}

\begin{abstract}
:
The article is framed within the context of the Doctoral Thesis Health Risk Factors in university students of pedagogy in Chilean universities, and it states the importance to watch over healthy styles and quality of life of the people in a formative environment, the meaning of a healthy university in the teacher training. As well as the social responsibility that these institutions have in which their students develop the necessary competences to face up health problems, the improvement of quality of life and the lack of habits and healthy life styles in the university population of the Chilean educational system in the present time and not only focused on the discipline domain.
\end{abstract}

Keywords: Healthy lifestyles - Quality of life - Healthy University.

I El artículo es resultado del proceso de estudios de doctorado del autor, realizados con el apoyo de la Beca Cardenal Raúl Silva Henríquez, obtenido en concurso desarrollado por la Universidad Católica Raúl Silva Henríquez.

* Dr. en Perspectivas Actuales de la Actividad Física y la Ciencia del Deporte de la Universidad de Las Palmas de Gran Canaria (España). Profesor de Estado en Educación Física Universidad de Chile. Egresado de Magíster en Administración en Gestión Deportiva U. Metropolitana de Ciencias de la Educación. Docente adscrito a la Universidad Católica Silva Henríquez.1valenzuela@ucsh.cl 


\section{Introducción}

El presente artículo tiene como uno de sus propósitos exponer la perspectiva de distintos autores respecto de lo que significan los estilos saludables y la calidad de vida de las personas, a partir de la atención sobre las consideraciones preliminares implicadas en el tema. Posteriormente, nos referimos al significado de una universidad saludable, destacando el rol que compete a las instituciones de educación superior en el apoyo e implementación de prácticas y estrategias para mejorar la salud y calidad de vida de sus estudiantes, docentes y funcionarios. Basado en el concepto de una ciudad saludable, que focaliza su atención en acciones de promoción de la salud orientadas al entorno de la comunidad universitaria, más que las estrategias de cambio de hábitos de cada persona en particular.

En este contexto, tenemos la convicción de que la mayor responsabilidad la tienen aquellas instituciones encargadas de la formación inicial docente, sin embargo otro agente importante de cambio es el profesor, que debiera, a nuestro entender, sentirse llamado a responder a este desafío. Para que además de formarlos expertos en la disciplinan también les entreguen las herramientas, para que actúen como modelos o promotores de conductas saludables a nivel de sus familias, en sus futuros entornos laborales y en la sociedad en general.

Seguidamente el artículo continúa con la exposición de los factores determinantes de la salud y los factores de riesgo para la salud en una comunidad universitaria. Finalizando este, con el significado de lo que es una escuela promotora de salud, conjuntamente con el repaso de algunas estrategias de promoción de la salud en las escuelas latinoamericanas y de Chile.

\section{Consideraciones preliminares}

Si bien los conceptos de "estilo de vida" y "calidad de vida" son usados con mucha frecuencia en los distintos ámbitos relacionados con la actividad y ejercicio físico, es necesario revisar inicialmente 
estos conceptos. En este sentido, Álvarez (2007) afirma que los estilos de vida saludable son procesos sociales, como así también las tradiciones, los hábitos, las conductas y las acciones de los individuos y conjuntos de personas, que conllevan a la satisfacción de los requerimientos del ser humano para alcanzar el bienestar y la vida.

El mismo autor señala que el concepto de "calidad de vida" es más difícil de determinar, principalmente por la dificultad para concretarlo, ya sea como una parte, o bien, como un todo.

En base a ello, Muñoz y Salinas (2004) expresan que no existe una teoría de calidad de vida que pueda dar cuenta de una definición para tal efecto. Los mismos autores hacen una revisión al planteamiento y proponen un conjunto de necesidades universales entre las que se destacan las siguientes:

- Subsistencia,

- Protección,

- Afecto,

- Entendimiento,

- Participación,

- Ocio,

- Creación,

- Identidad,

- Libertad.

Albala y col. (2004), exponen al respecto que la relación entre estas necesidades universales y su manera de satisfacerlas definirá la calidad de vida de cada persona, ya que ellas no dependen de la satisfacción de una sola necesidad aisladamente, sino que deben satisfacerse integradamente, de lo contrario la persona podría evidenciar deterioros en su calidad de vida.

Ahora bien, también es posible que cuando se obtiene satisfacción de una necesidad de forma aislada, dicho proceso estimule otras necesidades y colabore con ellas de manera simultánea, provocando una cadena en beneficio de la calidad de vida de una persona. 
Por tanto, el concepto de calidad de vida supone la satisfacción mínima aceptable del complejo de necesidades satisfactorias, en la dimensión individual y social, en los ámbitos locales y nacionales y cuidando la articulación entre lo micro y lo macro (Muñoz y Salinas, 2004).

Reforzando lo anterior, Max-Neef, Elizalde y Hopenhayn (1996) sostienen que una sociedad sana debe plantearse, como objetivo ineludible, el desarrollo de todas las personas y de toda la persona.

Por su parte Neogarten (1974), en Álvarez (2007), señala que la dicha de la vida contiene actividades diarias, el significado de la vida individual y colectivo, haciéndose responsable y partícipe de la misma; la percepción de logro de metas de vida, la autoimagen y la actitud hacia la vida. Sin embargo, para San Martín y Pastor (1988) la dicha de la vida es la respuesta a una interrelación de estímulos a nivel individual y social en un ambiente total, lo que nos lleva a concluir que dicha satisfacción se relaciona con los valores sociales que se practican.

En este contexto, el carácter integral de la salud humana y en esta perspectiva, los estilos de vida saludable, la actividad y ejercicios saludables deben tener una visión integral, en una dimensión colectiva y social que comprenda aspectos interrelacionados: el material, el social, el afectivo, el contexto sociocultural basado en lo ideológico, de cada persona y cada grupo que constituya una sociedad.

Un estilo de vida saludable, desde lo material, se caracteriza por las manifestaciones de la cultura material, vale decir, la vivienda, la alimentación y la ropa, entre otros. En lo social, en tanto, se definiría según las formas y estructuras organizativas, esto es: el tipo de familia, los grupos de parentesco, las redes sociales de apoyo y los sistemas de soporte tales como las instituciones y las asociaciones. Por último, en el plano ideológico los estilos de vida se demuestran por medio de las ideas, los valores y las creencias, las que, al fin y al cabo, son las que establecen las respuestas o conductas a los distintos acontecimientos de la vida. 
Una visión como ésta significa que, desde una perspectiva integral, los estilos de vida no debieran aislarse del contexto social, económico, político y cultural al cual pertenecen los individuos, sobre todo porque estos aspectos pueden influir positiva o negativamente en la salud de las personas.

Al respecto, Sánchez (1996) expresa que los estados positivos de salud están asociados a elementos tales como:

- Estilo de vida adecuado,

- Óptimo nivel de estrés,

- Sensación de bienestar,

- Capacidad de disfrute de la vida,

- Tolerancia a los retos que plantea el entorno.

Los estados negativos, en tanto, son todos aquellos conducentes al aumento de la morbilidad y que se asocian con problemas orgánicos (físicos, psicológicos y sociales), los que inciden prematuramente en la mortalidad de las personas.

Por su parte, Mendoza, Batista y Sagrera (1994) aseguran que en el seno de una sociedad establecida no hay un único estilo de vida saludable, sino muchos, independiente del concepto de salud que se acoja. "El estilo de vida saludable no existe, afortunadamente, porque implicaría sólo una cierta manera de afrontar diariamente la realidad en la que se vive. Entonces cualquier estilo de vida reconocido globalmente como saludable puede contener elementos no saludables, o saludables en un sentido pero no en otro” (p. I7).

En esta perspectiva, Sánchez (1996) manifiesta que es posible pensar que los hábitos de vida y de salud van estrictamente ligados, de forma que al darse esta relación debiéramos referirnos como concepto a hábitos saludables de vida, ya que conjuga, y parece hacer emerger el concepto de estilo de vida.

Seguidamente, Shephard (1994) expresa que al estudiar los estilos de vida se combinan una serie de comportamientos (hábitos 
positivos) que pueden afectar la salud de las personas, siendo tres de ellos fundamentales: alimentación adecuada, realización de actividades saludables y ejercicios saludables (ejecutados con frecuencia, intensidad, duración adecuada, con un volumen de carga, planificación adecuada a cada individuo y con un descanso regular aproximadamente de 7 a 8 horas).

En este mismo sentido, Shephard (1994) y Rodríguez (1995) consideran que entre los hábitos negativos para la salud se podrían mencionar los siguientes:

- Fumar cigarrillos

- Consumo excesivo de alcohol

- Comer en exceso o dietas inadecuadas

- Falta de realización de ejercicios saludables

\section{Universidades saludables}

Dado la presentación inicial en las consideraciones preliminares, se expone a continuación desde la perspectiva de Lange y Vio (2006) y otras instituciones de educación superior, actualmente existen en el país, con variadas experiencias en materia de promoción de la salud en las universidades, nos permitirá el primer acercamiento del significado de una universidad saludable.

Los autores señalan al respecto que la primera experiencia en estas materias convocó al Instituto de Nutrición y de Tecnología de Alimentos de la Universidad de Chile (INTA), a la Universidad de Concepción y a la Pontificia Universidad Católica de Chile. Una segunda iniciativa correspondió a la Red Universitaria de Protección y Autocuidado, que funciona desde 1999, desarrollando actividades para los estudiantes universitarios y de Educación superior del país acerca del uso y abuso de drogas. Por último, también destacan otras experiencias desarrolladas en el año 200I y que tuvieron como foco de atención el consumo de tabaco, alcohol y drogas en la Universidad Austral, Católica del Norte, de La Frontera, de Concepción y de 
Valparaíso, iniciativa motivada por las federaciones estudiantiles respectivas, el sistema de bienestar estudiantil y algunas facultades pertenecientes a dichas organizaciones.

Pese a ser plausible su esfuerzo, hay que destacar que estas iniciativas no obedecieron a políticas institucionales plasmadas en sus respectivos Planes de Desarrollo Estratégico, aunque contaron con la participación estudiantil, lo cual resulta relevante a la luz de su rol como agentes sociales y, particularmente en el caso de los estudiantes de distintas pedagogías, como futuros profesores del sistema educacional chileno.

Una Universidad Saludable o Promotora de Salud, se refiere a "aquella universidad que incorpora la Promoción de la Salud a su proyecto educativo y laboral, con el fin de favorecer el desarrollo humano y mejorar la calidad de vida de quienes estudian o trabajan y, a la vez, formarlos para que actúen como modelos o promotores de conductas saludables a nivel de sus familias, en sus futuros entornos laborales y en la sociedad en general" (Lange y Vio 2006, p. 9).

Así mismo entonces señalan los autores, que el concepto de Universidad Saludable o Institución de Educación Superior Saludable US/IESS se basa en los principios de "ciudades saludables" que priorizan iniciativas de promoción de la salud dirigidas al entorno universitario de manera global, más que las estrategias de cambio de hábitos de cada persona en particular. Este aspecto resulta vital, pues refuerza la idea de que el foco de la estrategia está en construir ambientes físicos, psíquicos y sociales que influyan en mejorar la calidad de vida de la comunidad universitaria, más que concentrarse en modificar los estilos de vida individuales. Este entorno será el estímulo para que las personas emprendan cambios positivos en su conducta. Desde esta perspectiva, quienes tienen la responsabilidad de la toma de decisiones en la estructura universitaria tienen la responsabilidad de proveer todas las condiciones para lograr estos ambientes que propendan a mejorar la calidad de vida universitaria. 
Las Instituciones de Educación Superior Promotoras de Salud son aquellas que se comprometen con la creación de un entorno y una cultura que fomente la salud y bienestar integral de los miembros que la componen: estudiantes, académicos y funcionarios. Para lograrlo, las universidades comprometidas con esta meta incorporan este concepto en sus políticas institucionales, en su entorno (lo que incluye las estructuras y procesos) y también en las mallas curriculares explícitas e implícitas.

El compromiso con la promoción de la salud se manifiesta actualmente en la universidad a través de sus múltiples roles: como organización educativa, como empleadora, como una institución comunitaria y frecuentemente como proveedora de servicios de salud. La universidad saludable viene a potenciar lo anterior, generando alianzas y apoyando iniciativas orientadas a mejorar el bienestar de su comunidad local y regional (Lange y Vio, 2006).

Complementariamente los mismos autores, nos plantean cinco aspectos o temas estratégicos a considerar en una universidad saludable:

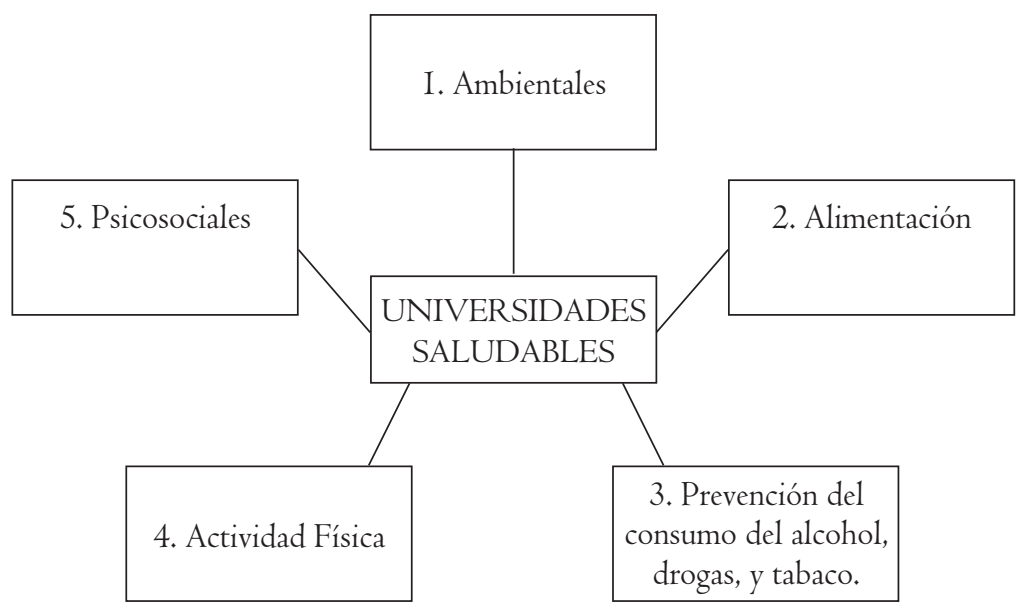

Cabe señalar, sin embargo, que algunas de estas estrategias no son compatibles con todos los establecimientos de educación superior, ya que su realidad en recursos (materiales, de infraestructura y humanos) esté muy lejana a lo sugerido. 
De este modo, desde la perspectiva psicosocial, una universidad saludable debe tener un entorno apropiado que permita relacionarse saludablemente, transformándose, primero que todo, en un factor facilitador para el aprendizaje de los estudiantes y de satisfacción laboral para los académicos y funcionarios, contribuyendo a aumentar el bienestar social y emocional de toda la comunidad universitaria. Los mismos autores señalan como características de los entornos psicosociales saludables los siguientes elementos:

- Ser cálidos, amistosos y que premian el aprendizaje

- Promueven la cooperación más que la competencia

- Promueven un estilo de comunicación abierta y directa

- Consideran importante dar oportunidad para la innovación y recreación

- Evitan la intimidación, el acoso y la violencia, a través del desarrollo de procedimientos y políticas que apoyen formas de mediación y resolución de conflictos en las aulas, entre los estudiantes y entre el personal y los estudiantes

- Promueven la igualdad de oportunidades y utilizan procedimientos democráticos para crear instancias y mecanismos de participación de los diferentes estamentos de la universidad

- Contribuyen a generar ambientes que influyan positivamente en la salud mental de la comunidad universitaria

Estas consideraciones se transforman, sin duda alguna, en acciones preventivas frente a estilos de vida poco saludables de la población universitaria, por ejemplo, respecto del consumo de drogas, alcohol y tabaco, así como también incitan al desarrollo de iniciativas promotoras de la salud, tales como la alimentación saludable, la realización de ejercicios saludables y de otros factores positivos como el manejo del estrés, la sexualidad responsable, el desarrollo de estilos de comunicación y la resolución de conflictos.

Por otra parte, los elementos ambientales consideran dos aspectos: el ambiente físico y el ambiente recreativo. 
- El ambiento físico representa la preocupación de una universidad saludable que se centra en mejorar y facilitar el acceso a un medio atractivo dentro de la universidad y que influye directamente en la calidad de vida de los estudiantes y de los que trabajan allí. Considerando tanto los terrenos de la propia universidad como los recursos materiales, de infraestructura y decorativos de la universidad (aulas, pasillos, cafetería, baños, oficinas, espacios para estudiantes, docentes y funcionarios). La inversión en los elementos ambientales debiera considerar, por ejemplo, senderos para caminar, ciclovías, facilitación de los accesos, seguridad en las escaleras, pisos antideslizantes, mobiliario ergonométrico y accesos para las personas discapacitadas.

- El ambiente recreativo, considera la preocupación por el fomento en la comunidad universitaria de diferentes actividades recreativas que se desarrollan en los espacios propios, o bien, en instalaciones externas, con el objeto de lograr la mayor participación del estudiante. Para eso es necesario considerar los gustos personales, las habilidades propias, y la influencia de compañeros, amigos y de algunos recursos como el costo, la disponibilidad de tiempo y el transporte.

Por otra parte, una alimentación equilibrada es necesaria para el bienestar y la armonía, no sólo desde el punto de vista energético sino que además mental y social. Una buena alimentación contribuye a potenciar los rendimientos académicos y laborales de una comunidad universitaria.

En relación con este aspecto y tal como revisáramos en puntos anteriores, la Organización Mundial de la Salud OMS (2006) considera que una alimentación compuesta por grasas saturadas, azúcar y sal, con baja ingesta de verduras y frutas, sumada a la ausencia de actividades y ejercicios saludables, son factores de riesgo para el desarrollo de enfermedades cardiovasculares, obesidad, diabetes, hipertensión y algunos tipos de cáncer.

Para contrarrestar estos efectos, en la comunidad universitaria se sugiere la implementación de acciones tales como: 
- La incorporación de actividades curriculares y contenidos implícitos y explícitos de alimentación saludable en los programas de pedagogía de forma transversal

- La promoción de cafeterías y casinos con alimentos saludables con precios similares a los de las comidas no saludables

- La supervisión de la variedad, calidad y sabor de los alimentos, así como también con los tiempos necesarios para consumirlos

Respecto de las actividades, una universidad saludable debe ser capaz de fomentar y generar ambientes y condiciones que promuevan estilos de vida activos, ya que al igual que el colegio, este es el lugar de mayor permanencia de los estudiantes durante el día, promoviendo por igual acciones como la realización de ejercicios y actividades saludables que ayuden a prevenir enfermedades y aumenten la productividad y el rendimiento académicos.

Finalmente, la prevención del consumo de alcohol, drogas y tabaco es un tema sensible para los estudiantes de Educación Superior, puesto que generalmente las acciones emprendidas en esta línea son restrictivas, punitivas y, en algunos casos, de ignorancia de las autoridades frente a la situación, manteniéndose alejadas de la responsabilidad de velar por la salud de los estudiantes. Algunos factores de riesgo de consumo de drogas ilícitas en el interior de una universidad son los siguientes:

- Baja autoestima

- Estrés académico, laboral o ambiental

- Disponibilidad de alcohol y drogas ilícitas y lícitas

- Escasez de programas de prevención y de ayuda permanente al estudiante

- Exigencias de rendimiento demasiado altas por parte de los docentes

\section{Factores determinantes de la salud}

Entonces si reconocemos los factores de riesgo del consumo de drogas, tabaco y alcohol es necesario a la vez identificar aquellos 
factores determinantes de la salud. En este contexto, Ramos (2003) indica que, desde la perspectiva de Lalonde (1974), consideraba la salud como una variable dependiente influida por la interacción de diferentes factores o determinantes, que se exponen a continuación:

El factor biológico o endógeno, apenas modificable, evidencia que los aspectos genéticos y los relativos al envejecimiento de las personas dependen de cada organismo y varían de una persona a otra. En el año 1974 la causa de muerte asociada a factores biológicos en los países desarrollados oscilaba entre el 10\% y el 25\%.

En relación con el factor ligado al medio ambiente, Peña (1990) señala que en el mismo período, los países desarrollados evidenciaban una causa de muerte asociada al medio ambiente que fluctuaba entre un $10 \%$ y $25 \%$. Este factor hace referencia a contaminantes físicos, químicos y biológicos, los cuales afectan sustantivamente la calidad de vida y la salud de las personas.

De los factores ligados a los estilos de vida, Mendoza y López (1993) señalan que los estilos de vida alcanzaban en igual época un $40 \%$ de las causas de muerte en los países desarrollados, en tanto que la suma del medio y los estilos de vida determinaban el 60\% y $80 \%$ de la mortalidad en países desarrollados. Así mismo, respecto a la mortalidad, Lalonde (1974), -en Ramos (2003)-, plantea que los factores ligados al sistema de asistencia sanitaria se encuentran entre un $10 \%$ y un $15 \%$ de las causas de muerte de los países avanzados. Asimismo los servicios sanitarios se representan por la cantidad, calidad, gratuidad y acceso a los mismos.

El informe de Lalonde (1974), que nos presenta Ramos (2003), pese a la antigüedad de los datos aportados, confirma la importancia de los estilos de vida como determinantes de una buena salud, siendo éstos cruciales para la realización de cambios en la gestión sanitaria de muchos países, los que tuvieron como resultado la entrega de mayores recursos para la promoción y la educación de la salud de su población. 


\section{Factores de riesgo para la salud en una comunidad universitaria}

Actualmente es evidente la preocupación permanente en la comunidad universitaria respecto de los estilos y hábitos de vida saludables de sus estudiantes. No obstante lo anterior "existen algunos que reconocidamente influyen sobre la sensación de bienestar y afectan la productividad laboral y académica” (Lange y Vio 2006, p. 22).

Ante esta situación, los mismos autores han identificado algunos factores de riesgo de la salud existentes en una comunidad universitaria, que identificamos a continuación.

- Sobrecarga académica o laboral: Se refiere a las dificultades que tiene un estudiante para adaptarse a la vida universitaria al salir del ámbito escolar, contexto que le exige rendimientos académicos, horarios y estilos de vida distintos de los que eran su costumbre, asumiendo una formación que por lo general no se basa en la persona, sino en el dominio de la disciplina.

- Falta de tiempo o de oportunidades para la actividad saludable, el esparcimiento y la alimentación saludable: $\mathrm{El}$ mismo proceso de adaptación y exigencias académicas provoca en los estudiantes dificultades para administrar su tiempo, que muchas veces no les alcanza para la realización de actividades saludables. Del mismo modo, los cambios de jornada no permiten contar con el tiempo necesario para alimentarse sanamente, o bien, los lugares para comer no incorporan alimentos saludables o sus espacios no son apropiados desde el punto de vista de la infraestructura que proveen, por lo que los estudiantes optan por almorzar comida rápida sentados en los patios o sencillamente en el suelo.

- Búsqueda de nuevas experiencias con el uso de alcohol, drogas y el inicio de la vida sexual: Las altas exigencias académicas, la necesidad de integración a ciertos grupos, el descubrir un mundo nuevo, sin control, la desorientación, sumados a una posible baja autoestima y autovaloración de sí mismos, puede llevar a los 
estudiantes a iniciarse en el consumo de estas sustancias o al inicio de una actividad sexual sin orientaciones sanitarias adecuadas.

- Exagerada autopercepción de buena salud de los jóvenes ("salud de hierro”): Ello implica que los jóvenes estudiantes viven su vida universitaria asumiendo, erróneamente, una salud que no siempre es la mejor, por lo que toman riesgos innecesarios para su cuidado y calidad de vida, sintiéndose fuertes y vitales sin serlo, muy probablemente.

- Inexistencia de espacios habilitados para fumadores: Al no contar con espacios habilitados para fumadores, los estudiantes utilizan cualquier lugar disponible, donde con frecuencia, conviven con estudiantes que no fuman. Al respecto, si bien la nueva Ley del consumo de tabaco en Chile se aplica desde el año 2006 en las instituciones de educación superior, la tendencia a utilizar los espacios disponibles por parte de los estudiantes fumadores continúa siendo recurrente.

\section{La promoción de la salud en las escuelas de Chile}

En la actualidad, el ámbito de la salud escolar en Chile se ha centrado en la modificación de los estilos de vida de su población. Ha sido un proceso largo en el que se ha abordado la prevención de enfermedades por medio de acciones estratégicas masivas y algunas recientes en promoción de salud, desde una perspectiva intersectorial.

Al respecto, Pezoa, Vidal y Guzmán (2004) manifiestan que Chile se encuentra en una etapa de término de una transición demográfica y epidemiológica, lo que implica que el estado de la salud en el país es comparable al de países desarrollados, donde los problemas de salud se relacionan con las enfermedades crónicas, salud mental y accidentes, con estilos de vida poco saludables, altos niveles de contaminación ambiental y un estado de inseguridad en la población.

Con estos antecedentes, el país dio inicio a un proceso de reformas tanto en el ámbito de la educación como en el de la salud, poniendo particular énfasis en el equilibrio entre la promoción y la prevención 
de acuerdo con las áreas que a cada uno le competía. Ahora bien, respecto de la Reforma educacional, resulta importante señalar dos elementos cruciales para la implementación de acciones tendentes a promover la salud en los establecimientos educativos: los objetivos transversales y el proyecto educativo de cada establecimiento (Pezoa y col. 2004).

En efecto, los objetivos transversales están orientados hacia la formación integral de la persona, y abarcan aspectos como la autoafirmación personal, que lleva a mejorar los niveles de autoestima y la manera en que los estudiantes se relacionan con otras personas. Por su parte, el proyecto educativo tiene una estricta relación con los objetivos transversales, los que a su vez están estrechamente relacionados con el concepto de establecimiento de educación promotor de salud.

Los autores complementan lo anterior, señalando que ante ambos escenarios, la promoción de la salud surge como una propuesta altamente efectiva y necesaria, pues busca que la comunidad educativa tome sus propias decisiones, concediendo herramientas para atender la salud, controlar el desarrollo de los factores de riesgo y potenciar los factores protectores.

Por su parte, el Ministerio de Salud de Chile, MINSAL (1998), en Pezoa y col. (2004), declaraba en la década de los '90 que la promoción fue una de las estrategias fundamentales para focalizarse en la calidad y en los estilos de vida saludables, los ambientes saludables y el desarrollo de valores en la comunidad. Lo anterior resulta muy relevante si consideramos que las escuelas son un espacio privilegiado para llevar a cabo acciones tendentes a promover la salud entre los niños y jóvenes, debido a su amplia cobertura (educación preescolar, básica y media).

En este mismo sentido, MINSAL (1999) señalaba que, de las escuelas promotoras de salud, los hábitos y conductas asociadas a una vida saludable tienen una mayor probabilidad de ser incorporados y de mantenerse a lo largo de la vida y que cada persona necesita también 
aprender a reconocer que posee la capacidad de modificar los factores que intervienen en su salud y en la de su comunidad.

Finalmente Pezoa y col. (2004), manifiestan que el Estado chileno creaba en 1999 el Consejo Nacional para la Promoción de Salud, llamado Vida Chile, el cual ha desarrollado un plan estratégico con metas y compromisos intersectoriales hasta el año 2010.

\section{Escuela promotora de salud}

Pezoa, Vidal y Guzmán (2004) señalan que una escuela promotora de salud debe ser aquella que realiza acciones integrales de promoción de la salud en torno al establecimiento educacional, como una ocasión para llevar estilos de vida saludables a la comunidad educativa y al entorno, favoreciendo el desarrollo humano de los estudiantes.

Lo anterior implica, necesariamente, el desarrollo de habilidades y destrezas para cuidar la salud y colaborar en el cuidado de la familia y de la comunidad, apropiándose críticamente de los saberes, competencias, actitudes y destrezas necesarias para comprender y transformar la realidad, formando personas creativas, seguras de sí mismas, críticas e innovadoras.

Además, el desarrollo de estas habilidades y destrezas propende a la promoción de valores, al fortalecimiento de la autoestima, la autovaloración, la asertividad y la emocionalidad, obteniendo de esta manera, la capacidad y las herramientas individuales necesarias para enfrentar las exigencias actuales con seguridad.

Todo lo anterior redundaría en un aumento de la capacidad productiva y del crecimiento espiritual de las personas, ya que se desarrollaría también la capacidad de amar, jugar, gozar y estimular los vínculos con otros, la integración y el crecimiento de la colectividad educativa, donde los estudiantes se constituyen de manera independiente, respetuosos de los derechos humanos. De acuerdo a lo planteado por los mismos autores, el profesor se transformaría en un motor fundamental de la promoción de la salud de sus estudiantes. 
Por otra parte, los mismos autores Pezoa y col. (2004), señalan que debido al aumento de enfermedades en niños y niñas en edad escolar en América Latina (obesidad, consumo de alcohol, tabaco y drogas, violencia, accidentes y embarazo en adolescentes), OPS ha difundido la iniciativa de las Escuelas Promotoras de Salud (EPS), con lo cual ha asumido, junto con la OMS, la misión de cooperar y colaborar técnicamente con otras regiones de América para establecer un consenso entre el sector educativo y el sector salud. Ello, con el propósito final de constituir alianzas con otros sectores, para conservar ambientes físicos y psicosociales saludables y desarrollar habilidades para alcanzar estilos de vida saludables en la comunidad educativa OPS/OMS (I996)

Para alcanzar estas metas, la OPS propuso tres ejes para la implementación de las EPS:

- Educación para la salud con un enfoque integral, es decir, considerando las necesidades y características individuales, de género y socioculturales de cada estudiante y fortaleciendo su autoestima.

- Creación de ambientes y entornos saludables, lo cual implica contar con espacios físicos limpios y amplios, áreas verdes, seguros, sin agresión física y/o verbal.

- Diseño y entrega de servicios de salud y alimentación, eje referido a la asistencia del estudiante al establecimiento educacional, asegurando la igualdad de oportunidades frente al aprendizaje OPS/OMS (I996).

Esta iniciativa de desarrollar y fortalecer la promoción y una educación para la salud en el ámbito escolar por la OPS, fue propuesta y aceptada en Costa Rica en el año 1993. En dicha oportunidad y consultados 12 representantes de los sectores de la salud y la educación, fueron identificadas las áreas que requerían mayor apoyo, formulándose posteriormente las recomendaciones a los Gobiernos miembros para implementarla. 
La estrategia de promoción y educación para la salud de la OPS/ OMS en el ámbito escolar hace referencia puntual al desarrollo y fortalecimiento para la salud de ambientes y entornos saludables, así como también de los servicios de salud y alimentación, los cuales comprometen a la escuela, la comunidad y a los servicios locales de salud, permitiendo emprender nuevas y mejores acciones para prevenir los riesgos de la salud en las futuras generaciones.

Para la OPS/OMS (1996) en Amador y Valenzuela (2010), señalan que estos compromisos se pueden traducir en los siguientes propósitos y acciones:

- Favorecer la formación de conocimientos, actitudes y prácticas de alimentación y salud en los alumnos y otros integrantes de la comunidad educativa.

- Proporcionar a los alumnos y alumnas las habilidades y destrezas indispensables para adoptar decisiones responsables en cuanto a la salud personal y que contribuyan al desarrollo y la seguridad de su entorno.

- Posibilitar el pleno desarrollo físico, psíquico, emocional y social, la autoestima y una imagen positiva de sí mismos en los alumnos y en toda la comunidad educativa.

- Favorecer modos de vida sanos y ofrecer a los alumnos y trabajadores de la educación, opciones de aprendizaje, experiencias, ambientes y entornos saludables.

- Desarrollar el sentido de responsabilidad y el compromiso de participación individual, familiar y social en acciones para mejorar la salud.

- Favorecer relaciones positivas y constructivas entre todos los miembros de la comunidad educativa y el cuidado de ambientes y entornos

- Ampliar la concepción de servicios de salud y alimentación para hacer de ellos un recurso educativo que apoye el aprendizaje y ayude a la comunidad a utilizar adecuadamente los sistemas y servicios de salud 
- Ofrecer un ambiente y marco de trabajo y estudio que promueva la salud, tomando en cuenta las condiciones del edificio, disponibilidad de agua, servicios de salud y alimentación, espacios deportivos y recreativos, y seguridad para todos.

- Definir objetivos claros de promoción de la salud y de seguridad para el conjunto de la comunidad escolar.

- Integrar la educación para la salud en el currículoa de forma coherente, utilizando metodologías que faciliten la participación de los alumnos en el proceso.

- Identificar y utilizar los recursos existentes en la comunidad para desarrollar acciones a favor de la promoción y la educación para la salud.

Lo fundamental de la propuesta es que se centra en los alumnos, en la comunidad, en el ambiente y en el entorno, optimizando los recursos, la integración en el currículo, la alimentación, las relaciones afectivas, la responsabilidad y el compromiso individual, familiar y social, es decir, las estrategias diseñadas están pensadas de una manera integral que considera todas las dimensiones del ser humano (cognitiva, emocional, física y social).

En la actualidad, la mayoría de los países miembros de la OPS/OMS cuentan con establecimientos educacionales en donde se realizan los diagnósticos para establecer e identificar ciertas anomalías en la salud en sus estudiantes, entre las cuales podemos destacar los siguientes problemas:

- De audición

- De visión

- Alteraciones posturales a nivel de columna, piernas y

- Salud bucal

- Higiene

- Alimentación

- Accidentes

- Saneamiento y otros (Amador y Valenzuela 2010) 
Al respecto, los autores señalan que en Costa Rica, El Salvador, Guatemala, Honduras, Nicaragua, Panamá, en el Caribe Hispano y en México, se cuenta con estos servicios para sus establecimientos escolares, pero al mismo tiempo se reconoce en ellos una falta de capacitación sistemática y poco actualizada de su personal docente, además de una insuficiencia en los materiales educativos que se utilizan para promover la salud. Esta situación es compartida por países como Bolivia, Colombia, Perú, Venezuela y Ecuador.

Este diagnóstico pone de manifiesto el desarrollo de la trayectoria de las escuelas en salud, destacándose el caso de Jamaica, país que realizó un programa para las escuelas primarias que incluyó estrategias comunicacionales y la creación de material didáctico innovador (poemas y tiras cómicas para maestros, por ejemplo).

Estados Unidos, por su parte, ha implementado un programa desde la escuela de párvulos hasta el decimosegundo grado. Como elementos destacables de esta iniciativa se encuentra el que cada escuela cuenta con una profesional en salud escolar capacitada (enfermera), además de incluir aspectos sobre promoción y prevención de enfermedades desde la Educación física hasta la Educación vial y de contar con profesores capacitados y apoyo de material didáctico.

Seguidamente, expresan los autores que en el hemisferio sur, en países como Chile, Argentina, Uruguay, Paraguay y Brasil se observa una Reforma educativa sin precedentes, declarándose que los servicios de salud son mayoritariamente asistenciales. En el caso de Chile, por ejemplo, dichos organismos señalan el aporte económico para extender los servicios básicos a un mayor número de escolares. Del mismo modo, se reconoce que esta Reforma educacional ha permitido incorporar contenidos básicos de salud, aun cuando existe la posibilidad de incorporarlos sólo de manera transversal, modalidad que presenta inconvenientes pues no es acreditable ni obligatoria, dejando su tratamiento a disposición del maestro.

Al respecto, la misma organización realizó un análisis crítico y estableció que la mayoría de los programas de educación en 
salud escolar tiene un enfoque vertical, utilizando una estrategia comunicacional unilateral para promover la salud en la escuela. Ante esta situación, lo recomendable sería fortalecer la capacidad institucional por medio de la implementación de una estrategia integral y con mayor amplitud.

Complementariamente, Amador y Valenzuela (2010) indican que un estudio comparativo de la OPS en conjunto con otras organizaciones, reveló que la mayoría de los países de la región enfrenta situaciones similares en la salud escolar, siendo coincidentes en cuanto a los contenidos, los enfoques educativos y la metodología de educación vertical, basándose además en los daños de la salud y en las causas de la enfermedad y muerte. Pese a ello, durante la última década, todos estos países han implementado programas de salud, proceso que no ha estado exento de dificultades, entre las que se cuentan:

- Falta de compromiso político.

- Falta de apoyo técnico continuo y coordinación entre los ministerios de Salud y Educación en todos los niveles.

- Insuficientes recursos nacionales en términos de: profesionales capacitados, capacitación a los maestros y materiales de capacitación.

- Carencia de mecanismos para dar seguimiento y evaluar programas.

- Falta de investigación acerca de las necesidades de la niñez y de la juventud para orientar los programas de salud escolar.

- Carencia de estrategias nacionales bien definidas para la promoción, apoyo, coordinación y administración de los programas de salud escolar.

- Falta de enfoques novedosos para el desarrollo de materiales educativos.

Es relevante detenerse en el punto que hace referencia a la capacitación de los maestros. Ello, porque si bien en todas las realidades educativas existen problemas como la insuficiencia de recursos materiales o de infraestructura, dificultades para implementar las políticas de 
Estado y ausencia de compromiso político o de compromiso de la propia unidad educativa, consideramos que la capacitación y las competencias de los profesores respecto de la salud estudiantil no debieran ser aspectos que quedaran al azar, sobre todo si se piensa en el importante rol que ellos tienen para la incorporación de hábitos y estilos de vida saludable en la población que atienden.

\section{Conclusiones}

Los estilos de vida saludable, la actividad y el ejercicio saludable, deben tener una visión global desde una dimensión colectiva y social, que comprenda aspectos interrelacionados: el material, el social, el afectivo y el sociocultural. No deben aislarse del contexto social, económico, político y cultural al cual pertenecen los individuos, sobre todo porque estos aspectos pueden influir positiva y negativamente en la salud de las personas.

Tanto la escuela como la universidad pueden y deben constituirse en organizaciones que incorporen prácticas y estrategias de promoción de salud, de acuerdo al contexto en el que se encuentran insertas, con la capacidad de transcender el aula llegando como un impacto positivo a su comunidad educativa, como incluso a nivel de país.

Las instituciones de educación superior promotoras de salud son aquellas que se comprometen con la creación de un entorno y una cultura que fomente la salud y el bienestar integral de los miembros que la componen: estudiantes, académicos y funcionarios.

La universidad saludable debe tener un entorno apropiado que permita relacionarse saludablemente, transformándose, ante todo, en un factor facilitador para el aprendizaje de los estudiantes y de satisfacción laboral para los académicos y funcionarios, contribuyendo a aumentar el bienestar social y emocional de toda la comunidad universitaria.

Las universidades comprometidas como promotoras de salud, deben incorporar acciones en sus políticas institucionales que emanen 
de su proyecto de planificación estratégica. Como así también en las propuestas curriculares de manera explícita e implícita, con la participación activa del equipo docente de la propia institución.

\section{Bibliografía}

Albala, C.; Olivares, S.; Salinas, J. y Vio, F. (2004). Bases, Prioridades y Desafíos de la Promoción de la Salud. Santiago, Chile: Universidad de Chile, Instituto de Nutrición y Tecnología de los Alimentos.

Álvarez, B. (2007). Prescripción del ejercicio, nuevo enfoque. Disponible en: http://www.una.ac.cr/maestria_salud/documents/prescripmaestria07.pdf

Amador, F. y Valenzuela, L. (2010). Hacia una Aptitud Deportiva Saludable. Santiago: Ediciones Universidad Católica Silva Henríquez.

Lange, I. y Vio, F. (2006). Guía para universidades saludables y otras instituciones de educación superior. Disponible en: http://www.minsal.cl/ici/ destacados/VerWUS.pdf

Max-Neef, M.; Elizalde, A. y Hopenhayn, M. (1996). Desarrollo a escala humana, una opción para el futuro. Devolopment Dialogue, $\mathrm{N}^{\circ}$ especial, 9-93.

Mendoza, R.; Batista, J. M. y Sagrera, M.R. (1994). Conductas Escolares Españolas Relacionadas con la Salud (1986-1990). Madrid: Consejo Superior de Investigaciones científicas.

Mendoza, R. y López, P. (1993). Escuelas generadoras de salud. Cuadernos de Pedagogía, 2l4, 8 - I2.

Ministerio de Salud, MINSAL (1999). Promoción de la Salud Para Chile. Santiago, Chile: Autor.

Muñoz., S. (2004). Promoción de la salud como Política de Estado. En Albalá, C.; Olivares, S.; Salinas, J. y Vio, F. (2004). Bases, Prioridades y Desafíos de la Promoción de la Salud. Santiago: Universidad de Chile.

Muñoz, F. y Salinas, J. (2004). Promoción de la salud como Política de Estado. En Albala, C.; Olivares, S.; Salinas, J. y Vio, F. (ed.), Bases, Prioridades y Desafíos de la Promoción de la Salud (pp. I-7). Santiago, Chile: Universidad de Chile, Instituto de Nutrición y Tecnología de Los Alimentos.

OMS (2006). Estrategia regional y plan de acción para un enfoque integrado sobre la prevención y el control de las enfermedades crónicas, incluyendo el régimen alimentario, la actividad física y la salud. Consultado el 9 
de diciembre de 2006. En http://www.paho.org/spanish/gov/cd/ CD47-I7rv-s.pdf

Organización Panamericana de la Salud y Organización Mundial de la Salud (1996). Escuelas promotoras de salud: modelo guía para la acción, Basado en la Experiencia Latinoamericana y Caribeña. Washington DC: Autores.

Peña, F. (I990). Salud ambiental. Madrid: Ciencia 3.

Pezoa, S.; Vidal, A. y Guzmán, M. (2004). Escuelas Promotoras de la Salud y Ambiente Laboral. En Albala, C.; Olivares, S.; Salinas, J. y Vio, F. (ed.), Bases, Prioridades y Desafíos de la Promoción de la Salud (pp. 240252). Santiago, Chile: Universidad de Chile, Instituto de Nutrición y Tecnología de los Alimentos.

Ramos, A. (2003). Actividad Física e Higiene para la Salud. Las Palmas de Gran Canaria: Universidad de Las Palmas de Gran Canaria.

Rodríguez, J. (1995). Psicología Social de la Salud. Madrid: Síntesis.

San Martín, H. y Pastor, V. (1988). Salud Comunitaria Teoría y Práctica. Madrid: Díaz de Santos.

Sánchez, F. (1996). La Actividad Física Orientada Hacia la Salud. Madrid: Biblioteca Nueva.

Shepard, R. (1994). Aerobic fitness \& health. Champaign: Human Kinetics. 